\title{
Nocturnal hypoxaemia after myocardial infarction: association with nocturnal myocardial ischaemia and arrhythmias
}

Søren Galatius-Jensen, Jan Hansen, Verner Rasmussen, Jesper Bildsøe, Mathias Therboe, Jacob Rosenberg

\begin{abstract}
Objective-To document the trend in arterial hypoxaemia and electrocardiographic abnormalities on the second to sixth nights after acute myocardial infarction.

Patients-Nineteen consecutive patients with acute myocardial infarction who

electrocardiographic abnormalities in most patients. Thus, episodic nocturnal hypoxaemia may be particularly detrimental to the infarcted myocardium in the early phase after infarction; special attention should therefore be directed towards oxygenation in this group of patients.
\end{abstract} were monitored continuously during the night (minimum 2300-0700) with a Holter tape recorder and a pulse oximeter. Fifteen patients were monitored for five nights, one patient for four nights, one patient for three nights, and two patients for two nights.

Results-Five patients had $>30$ episodic oxygen desaturations of $\geqslant 5 \%$ during the nights of monitoring and many patients had episodes with oxygen desaturations to $<80 \%$ ranging from $46 \%$ to $61 \%$ (from $7 / 15$ to $11 / 18$ patients) during the nights of monitoring. Constant hypoxaemia was found in 11-13\% (2/15) of the patients. Simultaneous episodic hypoxaemia and episodic tachycardia was seen in 9/17 $(52 \%)$ patients on the second night, 11/18 (61\%) on the third, $7 / 15(46 \%)$ on the fourth, 8/15 (53\%) on the fifth, and $5 / 15$ (33\%) on the sixth night. Simultaneous episodic hypoxaemia and ST deviation was seen in $5 / 17(29 \%)$ patients on the second night, $3 / 18(16 \%)$ on the third, $4 / 15$ $(26 \%)$ on the fourth, in no patients on the fifth, and in $3 / 5(20 \%)$ on the sixth night. Simultaneous occurrence of episodic hypoxaemia and arrhythmias (supraventricular, ventricular ectopy, and atrioventricular blockade) was seen in 5/17 $(29 \%)$ on the second night, $4 / 18(22 \%)$ on the third, $4 / 15(26 \%)$ on the fourth, $2 / 15$ (14\%) on the fifth, and in no patients on the sixth night. Overall, simultaneous occurrence of episodic hypoxaemia and electrocardiographic abnormalities (episodic tachycardia, ST deviations, and arrhythmias) was seen in 11/17 patients $(64 \%)$ on the second night, $13 / 18(72 \%)$ on the third, $10 / 15(66 \%)$ on the fourth, $8 / 15$ (53\%) on the fifth, and $7 / 15(46 \%)$ on the sixth night. One patient who died of cardiogenic shock had simultaneously occurring episodic hypoxaemia and nonsustained ventricular fibrillation on the night before she died.

Conclusion-Episodic and constant hypoxaemia are common during the first week after acute myocardial infarction. Episodic hypoxaemia was associated with
(Br Heart f 1994;72:23-30)

Despite improved treatment, mortality from acute myocardial infarction in hospital is still as high as $8-27 \% .^{1}$ Apnoeic episodes frequently occur in patients with congestive heart failure, ${ }^{23}$ chronic obstructive lung disease, ${ }^{45}$ and acute myocardial infarction, ${ }^{67}$ and they may cause episodic hypoxaemia and provoke arrhythmias and ischaemia. ${ }^{289} \mathrm{We}$ aimed to document the occurrence of nocturnal hypoxaemia in the subacute phase after myocardial infarction - that is, the second to sixth nights after infarction. Furthermore, we expected myocardium to be susceptible to hypoxaemia and we wanted to document a possible association between electrocardiographic abnormalities and nocturnal hypoxaemia during this time.

\section{Patients and methods}

We studied 19 consecutive patients admitted to our coronary care unit with myocardial infarction (table 1). Exclusion criteria were factors predisposing to hypoxaemia: lung disease requiring treatment, excessive obesity, scintigraphically detected pulmonary embolism, and symptomatic or objective signs of congestive heart failure.

All patients were monitored with a Holter

Table 1 Clinical and demographic date on 19 patients with acute myocardial infarction. Results are numbers of patients unless stated otherwise

\begin{tabular}{ll}
\hline Characteristic & \\
\hline Median age (range) (years) & $67(36-91)$ \\
No of men/women & $16 / 3$ \\
Median No of days in hospital (range) & $12(4-28)$ \\
No of smokers & 11 \\
Infarction site: & 9 \\
$\quad$ Anterior & 8 \\
Inferior & 2 \\
Septal & 5 \\
Maximum LDH (U/1): & 8 \\
<500 & 6 \\
$500-1500$ & 13 \\
1500-2500 & \\
\hline
\end{tabular}

$\mathrm{LDH}=$ lactate dehydrogenase. 
tape recorder and pulse oximetry. Patients were monitored for a total of five nights from the second to the sixth night after myocardial infarction; thus the acute phase was over. All nights were not of the same duration, but all patients completed eight hours of continuous monitoring on all study nights (2300-0700), and data from these eight hours were analysed.

\section{PULSE OXIMETRY}

Oxygen saturation was measured with a Nellcor N-200 (software version 2.7) pulse oximeter using an adhesive finger probe, ${ }^{10}$ with subsequent data printout on a chart recorder the following morning. The mean oxygen saturation during the night was calculated by estimating average values every 15 minutes throughout the night and taking an average of all the periods. ${ }^{11}$ Episodic hypoxaemia was defined as a decrease in oxygen saturation of $5 \%$ or more occurring within two minutes. ${ }^{11}$ These desaturations were classified into those with a lowest point equal to or greater than $80 \%$ oxygen saturation and those with a lowest point below $80 \%$. Minimum oxygen saturation was defined as the lowest single value for oxygen saturation obtained during the study period. Constant hypoxaemia was defined as a mean oxygen saturation of $<90 \%$ during the night in question. ${ }^{11}$

\section{HOLTER MONITORING}

Holter monitoring was peformed with an amplitude modulated, two channel tape recorder, either model ICR 7200 (Instruments for Cardiac Research, Liverpool, New York) or SpaceLabs model 90205 (SpaceLabs, Redmond, Virginia), with primary signal verification using a monitor (Quadriscope 8034, S and W Medico Teknik A/S, Copenhagen). The frequency response was 0.05 to $100 \mathrm{~Hz}(-3 \mathrm{~dB})$ and the tape speed $1 \mathrm{~mm} / \mathrm{s}$. Two separate bipolar leads were recorded: $\mathrm{CM}_{5}$ from electrodes placed in the second right intercostal space close to the sternal bone and in the $V_{5}$ position, and a modified orthogonal y lead from electrodes just to the left of the spinal column, one on the superior angle of the scapula and one above the iliac crest. ${ }^{12}$ The recordings were analysed semiautomatically with a computerised equipment (Pathfinder-3 professional (Reynolds Medical, Hertford)) or an Arrhythmiamaster $90101 \quad$ (SpaceLabs, Redmond, Virginia). All arrhythmic events detected during the automatic analyses were checked on a full disclosure report from both leads. Results of the ST segment analyses were presented in one-minute graphs from each lead. All episodes of ST segment deviation were verified by at least three strips from each (beginning, maximum, and end of deviation).

\section{CORRELATION BETWEEN}

ELECTROCARDIOGRAPHIC ABNORMALITIES

AND ARTERIAL HYPOXAEMIA

Precise information on simultaneous changes in myocardial arrthymias or ischaemia and oxygen desaturation was not possible because the recordings were made on two separate systems (pulse oximeter and Holter tape recorder). Synchronous time markers were placed at the start and end of each recording. Correlations between electrocardiographic abnormalities and oxygen desaturation were defined to exist if the electrocardiographic changes occurred within two minutes on either side of the oxygen desaturation.

\section{ETHICS}

The study was in accordance with the Helsinki declaration II and was approved by the local ethics committee. Patients were included if they gave their informed consent.

\section{STATISTICS}

Spearman's rank correlation test and Friedman's two way analysis by ranks were used for statistical analyses. Level of significance was chosen at $\mathrm{P}<0.05$.

\section{Results}

Tables 2, 3, and 4 summarise the results.

Nineteen patients were included in the study. Eighteen patients were monitored with pulse oximetry on the second night after infarction, 19 on the third night, 17 on the fourth night, 15 on the fifth night, and 16 on the sixth night. Eighteen patients were monitored with a Holter tape recorder on the second and third nights, 16 on the fourth and fifth nights, and 15 on the sixth night. The full monitoring protocol was not completed in one case because the patient died after the third nights, in two cases because the patients did not wish to participate any further, and in the remaining four cases because the patients did not complete a full eight hour monitoring of either pulse oximetry or Holter monitoring and were therefore excluded from the analyses.

\section{PULSE OXIMETRY}

Many patients seemed to have the sleep apnoea syndrome $(>30$ episodic oxygen desaturations of $\geqslant 5 \%$ during the night), the incidence ranging from $6-24 \%$ ( $1 / 18$ patient's to $4 / 17$ patients) and being lowest on the second night (table 2). Many patients had episodes with oxygen desaturations $<80 \%$, ranging from seven out of $15(46 \%)$ to 11 out of $18(61 \%)$ during the night of monitoring, with a median of 0-3 (range 0-41) episodes.

Two patients (cases 7 and 10) had constant hypoxaemia during the third to sixth and second to fourth nights, the patient in case 7 having a sinusoidal pattern during the whole night that is often seen in patients with Cheyne-Stokes respiration. Three patients (cases 9, 15, and 16) received supplemental oxygen as $100 \%$ oxygen through nasal cannulae. One patient (case 9 ) received $51 / \mathrm{min}$ on the second night, $2 \mathrm{l} / \mathrm{min}$ on the third night, and $2 \mathrm{l} / \mathrm{min}$ intermittently on the fourth night One patient (case 15) received oxygen at the rate $2 \mathrm{l} / \mathrm{min}$ from midnight on the fifth night, when he had an infarction, and at the 
Table 2 Electrocardiographic abnormalities and hypoxaemic events in individual patients. Numbers before colons indicate night of monitoring

\begin{tabular}{|c|c|c|c|}
\hline $\begin{array}{l}\text { Case } \\
\text { No }\end{array}$ & $\begin{array}{l}\text { No of nights with } \\
\text { monitoring }\end{array}$ & Electrocardiographic abnormalities & Hypoxaemic episodes \\
\hline 1 & $\begin{array}{l}\text { Holter } 5 \\
\text { Oximeter } 5\end{array}$ & $\begin{array}{l}\text { VPC index } 2: 2,3: 18,4: 20,5: 5,6: 10 \\
\text { ST depression } 2^{\star}, 3^{\star}, 4^{\star}, 5,6 \\
\text { Episodic tachycardia } 3^{\star}, 4^{\star}, 6^{\star} \\
\text { Non-sustained VT (few s) } 2^{\star} \\
\text { SVPCs } 3^{\star}, 4^{\star}\end{array}$ & $\begin{array}{l}\text { Episodic hypoxaemia with end point } \\
\geqslant 80 \% 2: 6,3: 21,4: 10,5: 13,6: 18 \\
<80 \% 2: 15,3: 0,4: 17,5: 0,6: 0\end{array}$ \\
\hline 2 & $\begin{array}{l}\text { Holter } 5 \\
\text { Oximeter } 5\end{array}$ & $\begin{array}{l}\text { VPC index } 2: 6,3: 7,4: 5,5: 6,6: 4 \\
\text { Episodic tachycardia } 2^{\star} \\
\text { SVPCs } 3^{\star}\end{array}$ & $\begin{array}{l}\text { Episodic hypoxaemia with end point } \\
\geqslant 80 \% 2: 4,3: 2,4: 1,5: 2,6: 5 \\
<80 \% 2: 1,3: 10,4: 17,5: 0,6: 1\end{array}$ \\
\hline 3 & $\begin{array}{l}\text { Holter } 5 \\
\text { Oximeter } 5\end{array}$ & $\begin{array}{l}\text { VPC index 2:4, 3:0, 4:0, 5:0, 6:3 } \\
\text { ST depression } 2,3\end{array}$ & $\begin{array}{l}\text { Episodic hypoxaemia with end point } \\
\geqslant 80 \% 2: 0,3: 1,4: 1,5: 6,6: 4 \\
<80 \% 2: 0,3: 0,4: 0,5: 0,6: 1\end{array}$ \\
\hline 4 & $\begin{array}{l}\text { Holter } 5 \\
\text { Oximeter } 5\end{array}$ & $\begin{array}{l}\text { VPC index } 2: 71,3: 68,4: 53,5: 60,6: 40 \\
\text { Episodic tachycardia } 2^{\star}, 3^{\star}, 4^{\star}, 5^{\star} \\
\text { Non-sustained SVT } 2^{\star}, 3^{\star}\end{array}$ & $\begin{array}{l}\text { Episodic hypoxaemia with end point } \\
\geqslant 80 \% 2: 3,3: 2,4: 31,5: 5,6: 5 \\
<80 \% 2: 4,3: 0,4: 4,5: 3,6: 3\end{array}$ \\
\hline 5 & $\begin{array}{l}\text { Holter } 5 \\
\text { Oximeter } 5\end{array}$ & $\begin{array}{l}\text { VPC index } 2: 1,3: 7,4: 10,5: 200,6: 25 \\
\text { ST depression } 3,4^{\star}, 5,6 \\
\text { Episodic tachycardia } 2,3,5 \\
\text { Non-sustained VT } 5^{\star}\end{array}$ & $\begin{array}{l}\text { Episodic hypoxaemia with end point } \\
\geqslant 80 \% \text { 2:7, 3:21, 4:34, 5:18, 6:30 } \\
<80 \% 2: 5,3: 4,4: 0,5: 2,6: 0\end{array}$ \\
\hline 6 & $\begin{array}{l}\text { Holter } 5 \\
\text { Oximeter } 5\end{array}$ & $\begin{array}{l}\text { VPC index } 2: 100,3: 146,4: 148,5: 116,6: 141 \\
\text { ST depression } 2^{\star}, 3^{\star}, 6 \\
\text { Episodic tachycardia } 2^{\star}, 3^{\star}, 4^{\star}, 5^{\star}, 6^{\star} \\
\text { Triplet VPCs } 4^{\star}, 5^{\star} \text { Isolated episodes }\end{array}$ & $\begin{array}{l}\text { Episodic hypoxaemia with end point } \\
\geqslant 80 \% 2: 21,3: 30,4: 5,5: 27,6: 25 \\
<80 \% 2: 0,3: 5,4: 2,5: 0,6: 0\end{array}$ \\
\hline 7 & $\begin{array}{l}\text { Holter } 5 \\
\text { Oximeter } 5 \\
\text { (nights 3-6) }\end{array}$ & $\begin{array}{l}\text { VPC index } 2: 4,3: 2,4: 1,5: 0,6: 2 \\
\text { ST depression } 6^{\star} \\
\text { Episodic tachycardia } 3^{\star}, 4^{\star}, 5^{\star}\end{array}$ & $\begin{array}{l}\text { Episodic hypoxaemia with end point } \\
\geqslant 80 \% 3: 320,4: 250,5: 330,6: 290 \\
<80 \% 3: 163,4: 0,5: 4,6: 5 \\
\text { Constant hypoxemia } 3,4,5,6\end{array}$ \\
\hline 8 & $\begin{array}{l}\text { Holter } 5 \\
\text { Oximeter } 5\end{array}$ & $\begin{array}{l}\text { VPC index } 2: 8,3: 3,4: 4,5: 0,6: 3 \\
\text { ST depression } 2,3,4,6 \\
\text { Episodic tachycardia } 3^{\star}\end{array}$ & $\begin{array}{l}\text { Episodic hypoxaemia with end point } \\
\geqslant 80 \% 2: 1,3: 2,4: 6,5: 25,6: 8 \\
<80 \% 2: 0,3: 1,4: 0,5: 0,6: 0\end{array}$ \\
\hline 9 & $\begin{array}{l}\text { Holter } 5 \\
\text { Oximeter } 5\end{array}$ & $\begin{array}{l}\text { VPC index } 2: 0,3: 2,4: 0,5: 1,6: 0 \\
\text { Episodic tachycardia } 3^{\star}, 5^{\star} \\
\text { Triplet VPCs } 2^{\star} \\
\text { AV blockade } 3^{\star}\end{array}$ & $\begin{array}{l}\text { Episodic hypoxaemia with end point } \\
\geqslant 80 \% 2: 0,3: 18,4: 18,5: 2,6: 19 \\
<80 \% 2: 0,3: 0,4: 0,5: 0,6: 0 \\
\text { Supplemental oxygen } 2,3,4\end{array}$ \\
\hline 10 & $\begin{array}{l}\text { Holter } 5 \\
\text { Oximeter } 5\end{array}$ & $\begin{array}{l}\text { VPC index } 2: 2,3: 0,4: 0,5: 0,6: 0 \\
\text { ST depression } 2^{\star}, 3^{\star}, 4^{\star}, 5,6 \\
\text { Episodic tachycardia } 6^{\star}\end{array}$ & $\begin{array}{l}\text { Episodic hypoxaemia with end point } \\
\geqslant 80 \% 2: 7,3: 4,4: 4,5: 6,6: 9 \\
<80 \% 2: 0,3: 0,4: 0,5: 0,6: 0 \\
\text { Constant hypoxaemia } 2,3,4\end{array}$ \\
\hline 11 & $\begin{array}{l}\text { Holter 3 } \\
\text { (nights 2-4) } \\
\text { Oximeter } 5\end{array}$ & $\begin{array}{l}\text { VPC index } 2: 7,3: 4,4: 10 \\
\text { Episodic tachycardia } 2^{\star}, 3^{\star}\end{array}$ & $\begin{array}{l}\text { Episodic hypoxaemia with end point } \\
\geqslant 80 \% 2: 10,3: 9,4: 10,5: 1,6: 4 \\
<80 \% 2: 10,3: 0,4: 0,5: 0,6: 15\end{array}$ \\
\hline 12 & $\begin{array}{l}\text { Holter } 4 \\
\text { (nights 2, 3, 5, 6) } \\
\text { Oximeter 5 }\end{array}$ & $\begin{array}{l}\text { VPC index } 2: 2,3: 0,5: 0,6: 1 \\
\text { ST depression } 2 \\
\text { Episodic tachycardia } 3^{\star}\end{array}$ & $\begin{array}{l}\text { Episodic hypoxaemia with end point } \\
\geqslant 80 \% 2: 10,3: 9,4: 10,5: 1,6: 4 \\
<80 \% 2: 10,3: 0,4: 0,5: 0,6: 1\end{array}$ \\
\hline 13 & $\begin{array}{l}\text { Holter } 5 \\
\text { Oximeter } 5\end{array}$ & $\begin{array}{l}\text { VPC index 2:0, 3:1, 4:0, 5:0, 6:0 } \\
\text { ST depression } 2-5 \\
\text { Episodic tachycardia } 4^{\star}, 5^{\star} \\
\text { SVPC } 4^{\star}\end{array}$ & $\begin{array}{l}\text { Episodic hypoxaemia with end point } \\
\geqslant 80 \% 2: 1,3: 4,4: 19,5: 22,6: 25 \\
<80 \% 2: 10,3: 0,4: 0,5: 1,6: 18\end{array}$ \\
\hline 14 & $\begin{array}{l}\text { Holter } 5 \\
\text { Oximeter } 5\end{array}$ & $\begin{array}{l}\text { VPC index } 2: 0,3: 0,4: 0,5: 0,6: 0 \\
\text { ST depression } 2^{\star}, 3,4^{\star}, 5,6^{\star} \\
\text { Episodic tachycardia } 2^{\star}, 3^{\star}, 5^{\star}, 6^{\star}\end{array}$ & $\begin{array}{l}\text { Episodic hypoxaemia with end point } \\
\geqslant 80 \% 2: 62,3: 57,4: 52,5: 52,6: 62 \\
<80 \% 2: 17,3: 41,4: 5,5: 10,6: 17\end{array}$ \\
\hline 15 & $\begin{array}{l}\text { Holter } 5 \\
\text { Oximeter } 5\end{array}$ & $\begin{array}{l}\text { VPC index } 2: 420,3: 360,4: 200,5: 48,6: 4 \\
\text { ST depression } 5,6^{\star} \\
\text { Episodic tachycardia } 2^{\star}, 4^{\star}, 5^{\star}, 6^{\star} \\
\text { Bigemini } 2^{\star} \\
\text { SVPC } 4^{\star} \\
\text { Torsade de pointes, reinfarction } 5\end{array}$ & $\begin{array}{l}\text { Episodic hypoxaemia with end point } \\
\geqslant 80 \% 2: 4,3: 10,4: 18,5: 13,6: 10 \\
<80 \% 2: 3,3: 30,4: 20,5: 8,6: 0 \\
\text { Supplemental oxygen } 5,6\end{array}$ \\
\hline 16 & $\begin{array}{l}\text { Holter } 5 \\
\text { Oximeter } 5\end{array}$ & $\begin{array}{l}\text { VPC index 2:4, 3:60, 4:30, 5:64, } 6: 0 \\
\text { ST depression } 2-6 \\
\text { Episodic tachycardia } 2^{\star}, 4^{\star}\end{array}$ & $\begin{array}{l}\text { Episodic hypoxaemia with end point } \\
\geqslant 80 \% 2: 10,3: 18,4: 29,5: 15,6: 20 \\
<80 \% 2: 0,3: 1,4: 1,5: 7,6: 6 \\
\text { Supplemental oxygen } 2\end{array}$ \\
\hline 17 & $\begin{array}{l}\text { Holter } 2 \\
\text { (nights 2-3) } \\
\text { Oximeter } 3 \\
\text { (nights 2-4) }\end{array}$ & $\begin{array}{l}\text { VPC index } 2: 0,3: 2 \\
\text { ST depression } 2 \star, 3 \\
\text { Episodic tachycardia } 2,3\end{array}$ & $\begin{array}{l}\text { Episodic hypoxaemia with end point } \\
\geqslant 80 \% 2: 25,3: 26,4: 2 \\
<80 \% 2: 2,3: 10,4: 0\end{array}$ \\
\hline 18 & $\begin{array}{l}\text { Holter 1 } \\
\text { (night 3) } \\
\text { Oximeter } 2 \\
\text { (nights 2-3) }\end{array}$ & $\begin{array}{l}\text { VPC index } 3: 755 \\
\text { Episodic tachycardia } 3^{\star} \\
\text { Non-sustained VT } 30 \mathrm{~s} 3^{\star}\end{array}$ & $\begin{array}{l}\text { Episodic hypoxaemia with end point } \\
\geqslant 80 \% 2: 5,3: 30 \\
<80 \% 2: 11,3: 9 \\
\text { Died during daytime after night } 3\end{array}$ \\
\hline 19 & $\begin{array}{l}\text { Holter 1 } \\
\text { (night 2) } \\
\text { Oximeter 2 } \\
\text { (nights 2-3) }\end{array}$ & VPC index $2: 2$ & $\begin{array}{l}\text { Episodic hypoxaemia with end point } \\
\geqslant 80 \% 2: 18,3: 20 \\
<80 \% 2: 2,3: 0\end{array}$ \\
\hline
\end{tabular}

VPC = ventricular premature contraction, VT = ventricular tachycardia, SVT = supraventricular tachycardia,

SVPC = supraventricular premature contraction.

^Episodic hypoxaemia and electrocardiographic events occurring within two minutes of each other. ST deviations $\geqslant 0 \cdot 1 \mathrm{mV}$ were defined as significant. Episodic tachycardia was defined as an episodic increase in heart rate of $10 \%$ or more from baseline. Episodic oxygen desaturation was defined as a decrease in oxygen saturation of $5 \%$ or more occurring within two minutes. Constant hypoxaemia was defined as a mean saturation $\leqslant 90 \%$ on given night.

rate of $2 \mathrm{l} / \mathrm{min}$ on the sixth night. Another patient (case 16 ) received oxygen at a rate of $2 \mathrm{l} / \mathrm{min}$ on the second night. Severe hypoxaemia (oxygen saturation $=\leqslant 85 \%$ ) was seen for shorter lengths of time in three patients on the second night (in case 4 saturation was
$80 \%$ for 15 minutes, in case 9 saturation was $84 \%$ for 40 minutes, and in case 15 saturation was $85 \%$ for 60 minutes). On later nights no periods of oxygen saturation of $\leqslant 85 \%$ were detected that lasted for more than two minutes. 
Table 3 Frequency of electrocardiographic abnormalities and hypoxaemic events

\begin{tabular}{|c|c|}
\hline & $\begin{array}{l}\text { Night of monitoring: } \\
\text { No or proportion of patients }\end{array}$ \\
\hline $\begin{array}{l}\text { No of patients monitored } \\
\text { No of patients with: } \\
\text { ST deviation } \\
\text { ST deviation } \\
\text { Tachycardia } \\
\text { Arrhythmias } \\
\text { ECG abnormalities (total) }\end{array}$ & $\begin{array}{l}\text { ing data } \\
2: 18,3: 18,4: 16,5: 16,6: 15 \\
2: 10 / 18,3: 10 / 18,4: 7 / 16,5: 7 / 16,6: 9 / 15 \\
2: 5 / 18,3: 3 / 18,4: 4 / 16,5: 0 / 16,6: 4 / 15 \\
2: 18 / 18,3: 11 / 18,4: 7 / 16,5: 8 / 16,6: 6 / 15 \\
1: 7 / 18,2: 4 / 18,3: 11 / 16,4: 3 / 16,5: 0 / 15 \\
2: 11 / 18,3: 12 / 18,4: 9 / 16,5: 9 / 16,6: 7 / 15\end{array}$ \\
\hline $\begin{array}{l}\text { No of patients monitored } \\
\text { No of patients with constant hypoxaemia } \\
\text { No of patients with hypoxaemic episodes } \\
\text { with end point } \geqslant 80 \% \\
\text { No of patients with hypoxaemic episodes } \\
\text { with end point }<80 \% \\
\text { No of patients with }>30 \text { hypoxaemic episodes } \\
\text { (regardless of end point) }\end{array}$ & $\begin{array}{l}\text { try data } \\
2: 18,3: 19,4: 17,5: 15,6: 16 \\
2: 2 / 18,3: 2 / 19,4: 2 / 17,5: 2 / 15,6: 2 / 16 \\
2: 16 / 18,3: 19 / 19,4: 17 / 17,5: 15 / 15,6: 16 / 16 \\
2: 11 / 18,3: 11 / 19,4: 9 / 17,5: 7 / 15,6: 9 / 16 \\
2: 1 / 18,3: 4 / 19,4: 4 / 17,5: 2 / 16,6: 3 / 16\end{array}$ \\
\hline
\end{tabular}

Table 4 Medians (ranges) of individual mean values from pulse oximetry and electrocardiography on the second to sixth nights after acute myocardial infarction

\begin{tabular}{llllll}
\hline \multicolumn{5}{c}{ Night after infarction } \\
\cline { 2 - 6 } & 2 & 3 & 4 & 5 & 6 \\
\hline Oxygen saturation (\%): & $94(90-98)$ & $93(87-98)$ & $93(89-97)$ & $94(89-98)$ & $94(88-97)$ \\
$\quad$ Mean & $79(45-93)$ & $79(45-92)$ & $82(45-90)$ & $81(47-91)$ & $80(55-91)$ \\
$\quad \begin{array}{l}\text { Minimum } \\
\text { No of hypoxaemic episodes: }\end{array}$ & $6(0-62)$ & $18(1-320)$ & $10(0-250)$ & $13(1-330)$ & $19(4-290)$ \\
$\geqslant 80 \%^{\star}$ & $3(0-17)$ & $1(0-41)$ & $0(0-20)$ & $0(0-10)$ & $1(0-17)$ \\
$<80 \%$ & $4(0-755)$ & $3(0-366)$ & $5(0-200)$ & $1(0-200)$ & $3(0-141)$ \\
VPC index & $99(74-173)$ & $94(56-120)$ & $91(69-123)$ & $94(69-163)$ & $94(71-151)$ \\
Heart rate: & $59(45-75)$ & $60(39-80)$ & $59(46-78)$ & $59(45-74)$ & $58(44-75)$ \\
Maximum & $73(61-107)$ & $72(59-94)$ & $67(53-91)$ & $75(52-88)$ & $73(54-100)$ \\
Minimum & Mean & &
\end{tabular}

VPC $=$ No of ventricular premature beats per hour of monitoring.

${ }^{\star} \mathrm{P}<0.05$ by Friedman test.

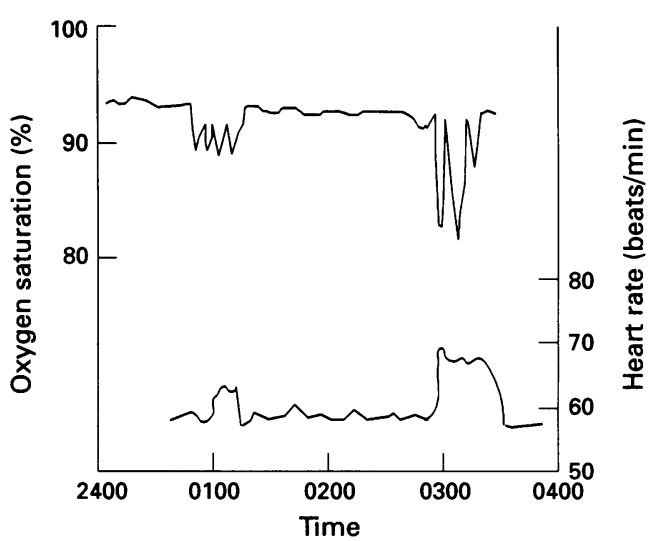

Figure 1 Simultaneously occurring hypoxaemia and episodic tachycardia in case 6 on third night after infarction.

aemia and episodic tachycardia (increase in heart rate of more than $10 \%$ from baseline) (figure 1) ranged from $33 \%(5 / 15)$ (sixth night) to $61 \%(11 / 18)$ (third night) of the patients. Simultaneous occurrence of episodic hypoxaemia and ST deviation (figure 2) ranged from 0 (fifth night) to $29 \%(5 / 17)$ (second night) of the patients. Simultaneous occurrence of episodic hypoxaemia and arrhythmias (supraventricular, ventricular, or atrioventricular blockade) (figures 3 and 4) was seen in ranging from 0 (sixth night) to $29 \%(5 / 17)$ (second night) of the patients. On the second night we found a significant correlation between maximum heart rate and minimum oxygen saturation $\left(r_{\mathrm{s}}=-0.54, \mathrm{P}=\right.$ 0.03 ) and between maximum heart rate and number of oxygen desaturations with a minimum value below $80 \%\left(r_{\mathrm{s}}=0.14, \mathrm{P}=0.004\right)$; we found no significant correlation between maximum heart rate and mean oxygen saturation during the same night $(P=0.09)$. In summary, the simultaneous occurrence of episodic hypoxaemia and electrocardiographic abnormalities (episodic tachycardia, ST deviation, and arrhythmias) ranged from $46 \%$ (7/15) (sixth night) to $72 \%(13 / 18)$ (third night) of the patients.

One patient (case 18) died of cardiogenic shock during the day after the third night. She had one episode of non-sustained ventricular tachycardia (22 s) and simultaneously occurring episodic hypoxaemia (oxygen saturation $70 \%$ for one minute) on the night before she died (figure 4).

he showed a $0.3 \mathrm{mV}$ ST elevation in an electrocardiogram, but at no time did oxygen saturation fall below $90 \%$. One patient (case 18) had an index of 755 on the night before she died of cardiogenic shock. Electrocardiographic abnormalities did not coincide with episodic hypoxaemia in two cases: one patient (case 3) had an ST depression on the second and third nights and another (case 19) had no electrocardiographic abnormalities on the second night, when he wore the Holter monitor.

\section{HYPOXAEMIA LINK WITH}

ELECTROCARDIOGRAPHIC ABNORMALITIES

Tables 2, 3 and 4 summarise the results. Simultaneous occurrence of episodic hypox-

\section{Discussion}

We found a high incidence of episodic hypoxaemia and a possible association between episodic hypoxaemia and electrocardiographic abnormalities in most patients with acute myocardial infarction.

Previous studies have shown a high incidence of sleep apnoea in patients with myocardial infarction. ${ }^{67}$ Several studies have shown coincidence between arrhythmias, ischaemia, and hypoxaemia in patients with left heart failure, ${ }^{23}$ with the sleep apnoea syndrome, ${ }^{89}$ and after major surgery, ${ }^{11}$ and these findings have now been confirmed in the sub- 
Figure 2 Simultaneously occurring ST depression and hypoxaemia in case 6 on second night and in case 14 on fourth night after infarction.
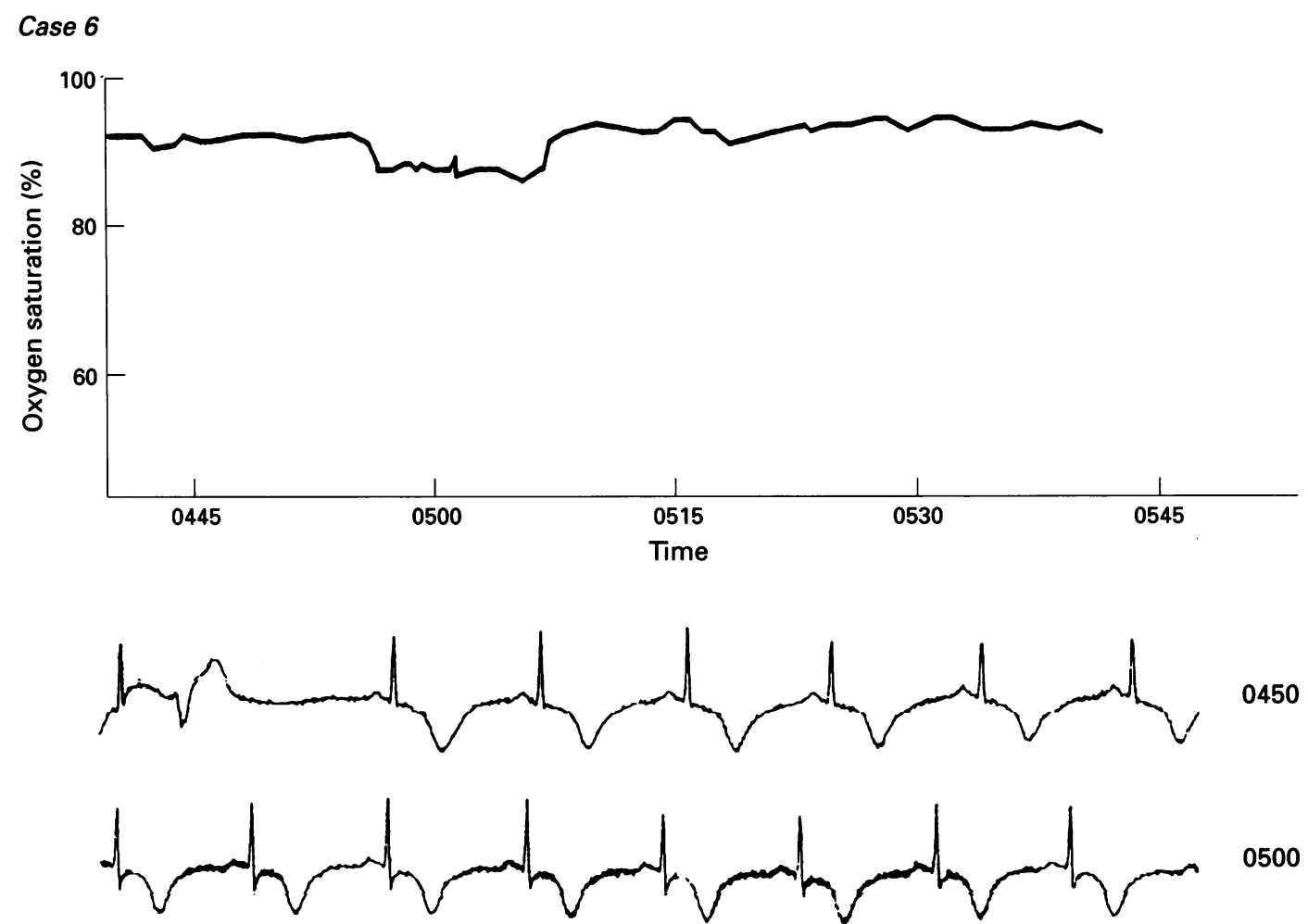

0500

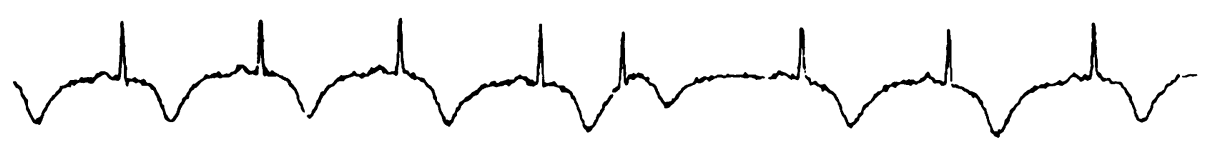

0520

Time

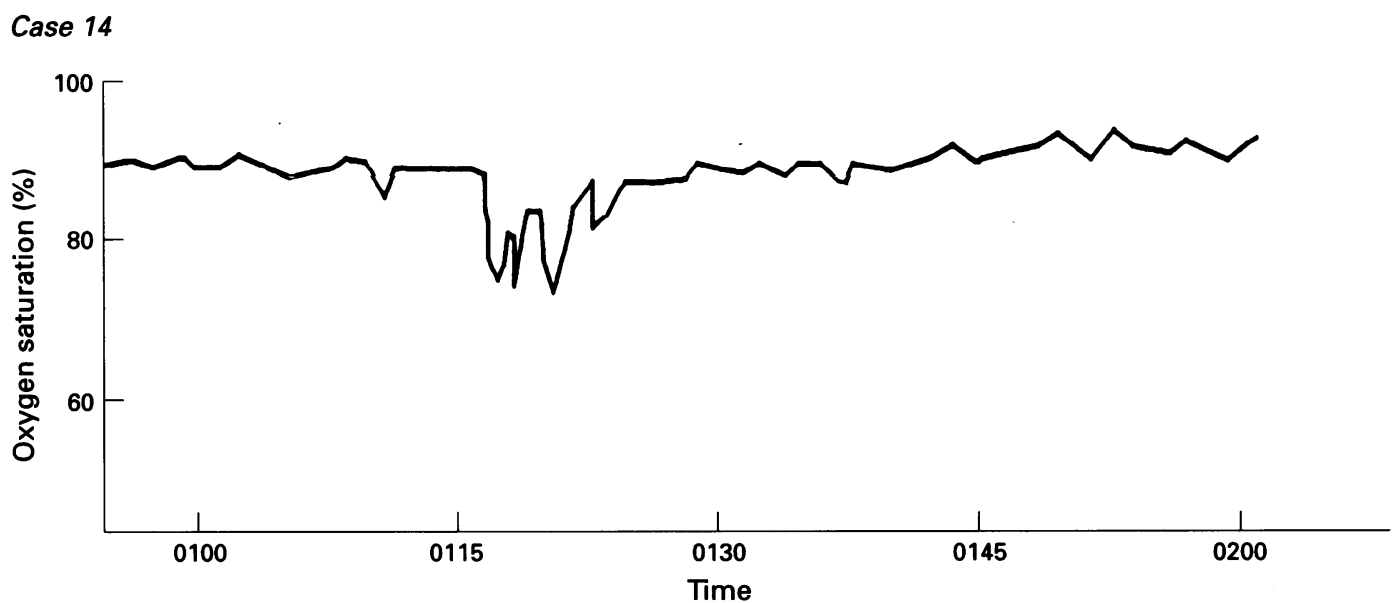

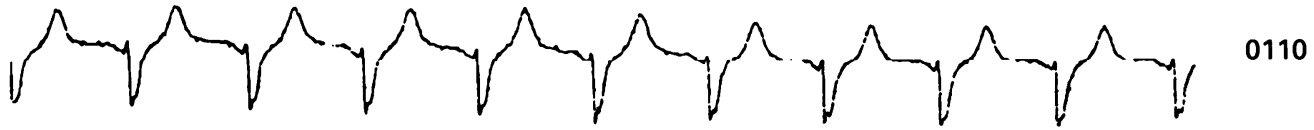

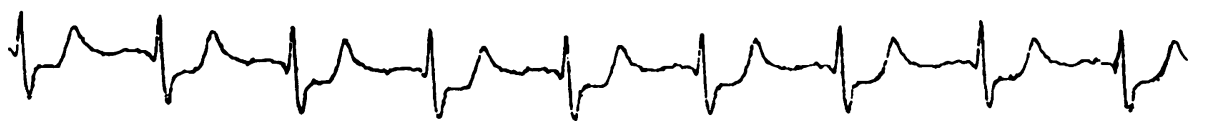

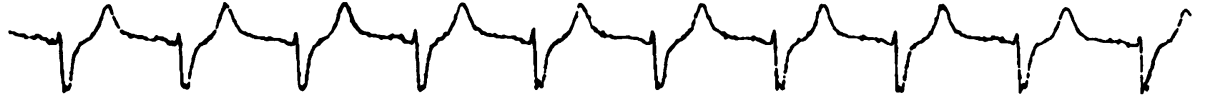


Figure 3 Simultaneously occurring non-sustained supraventricular third nights after infarction in case 4. tachycardia and

hypoxaemia on second and

Second night
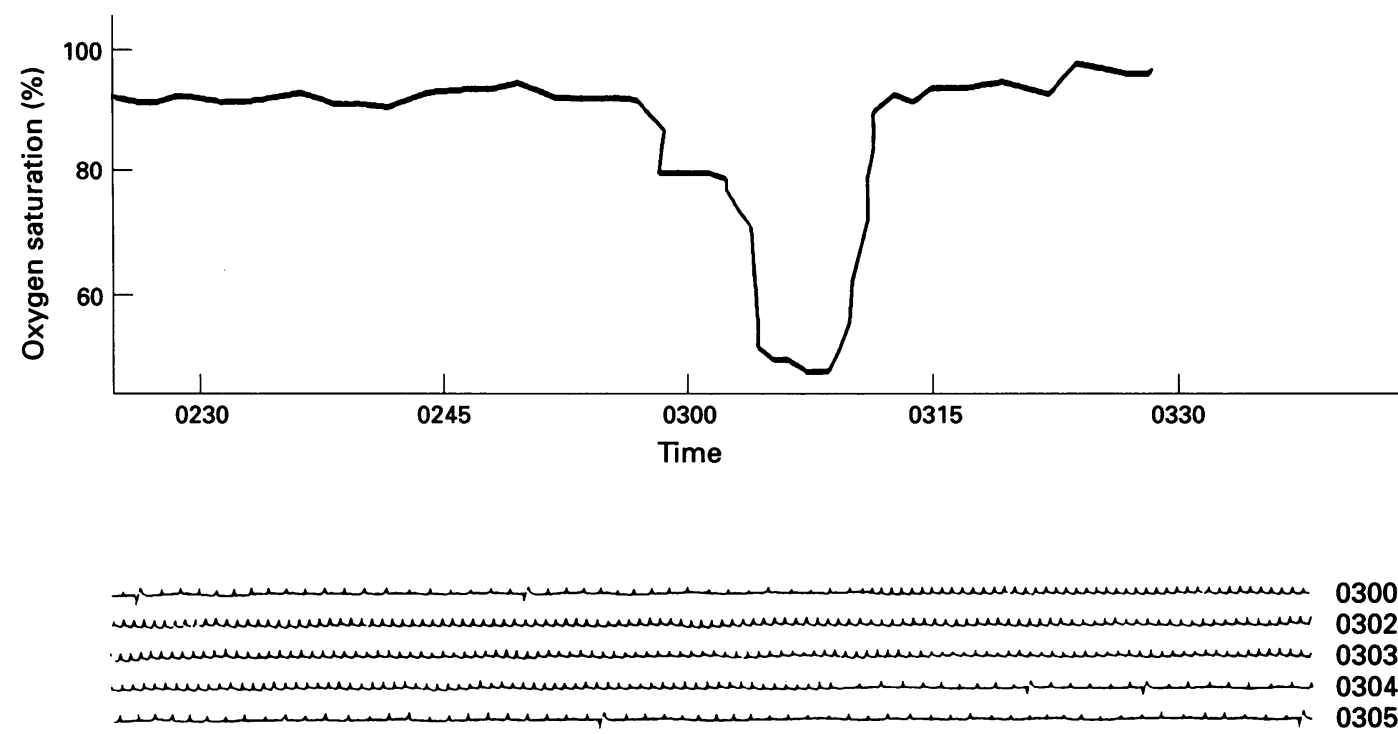

Time
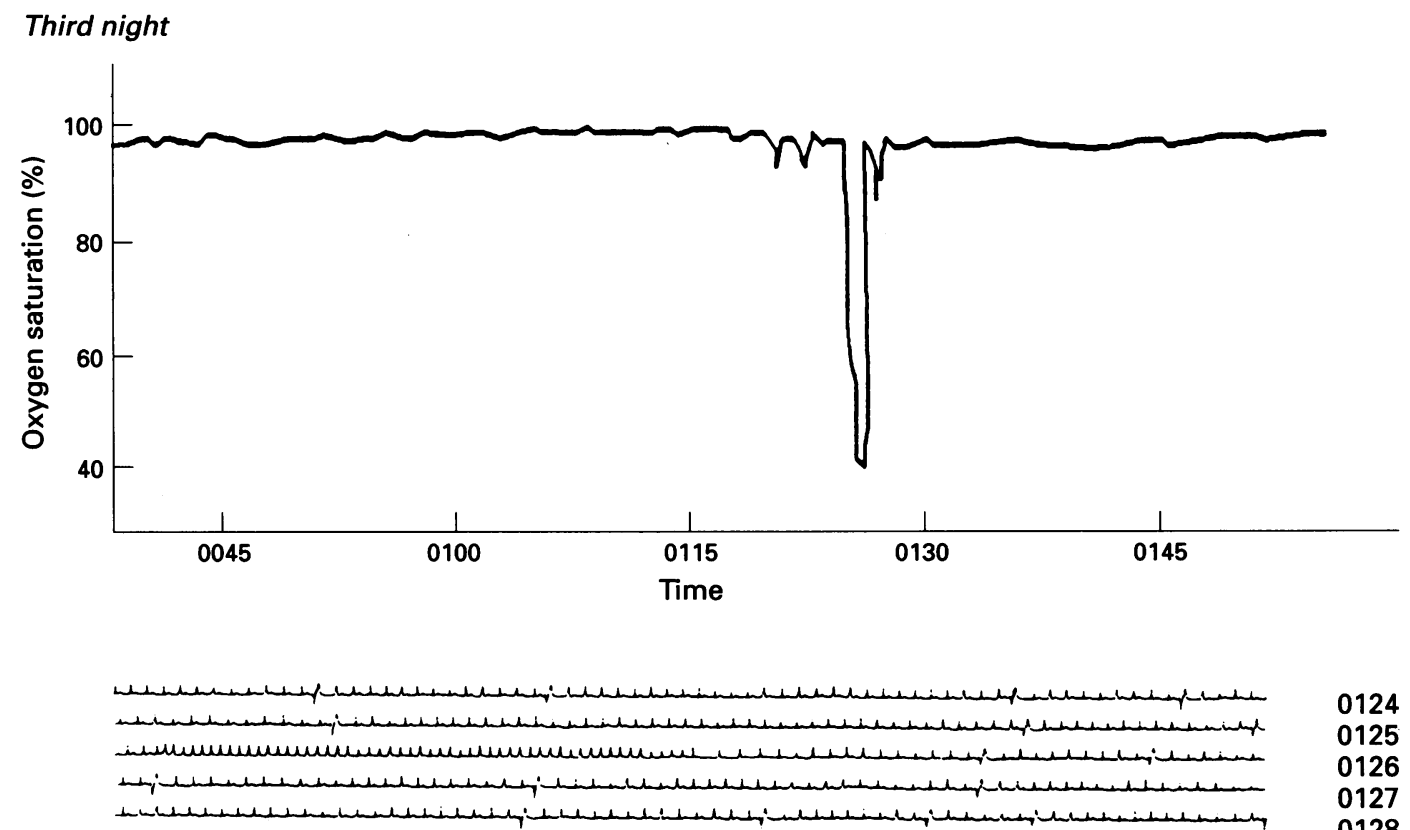

Figure 4 Simultaneously occurring non-sustained ventricular tachycardia and hypoxaemia in case 18 on third night after infarction. Patient died on following day of cardiogenic shock.

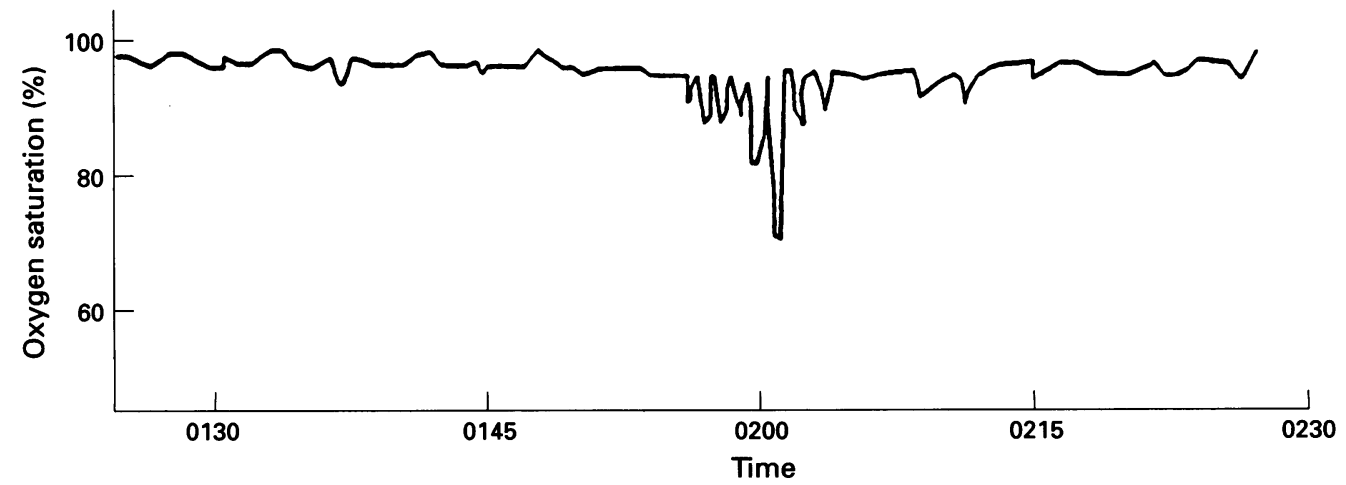

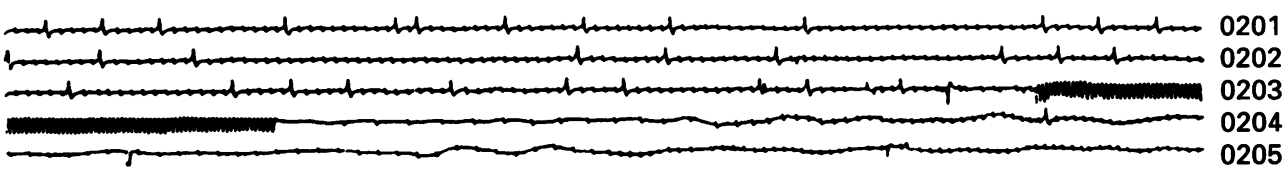


acute stage after acute myocardial infarction To our knowledge, ours is the first study to show the incidence of constant and episodic nocturnal hypoxaemia in the subacute stage after myocardial infarction and the association between hypoxaemia and electrocardiographic abnormalities by continuous monitoring during the first nights after an infarction.

Some of the episodes of hypoxaemia observed during monitoring by pulse oximetry might be artefacts, the result of the probe moving or being displaced. ${ }^{13}$ A study in patients with sleep apnoea or lung disease showed, however, that more than $99 \%$ of the observed episodes of desaturation were reflected in transcutaneous oxygen tensions, ${ }^{14}$ suggesting that overnight pulse oximetry gives a reliable estimate of episodic hypoxaemia. Furthermore, in our study the probe was secured with adhesive tape, making displacement and therefore artefacts highly unlikely. Finally, the findings of simultaneously occurring episodic hypoxaemia and electrocardiographic abnormalities when obtained by two different methods of measurement (pulse oximetry and Holter monitoring) verify that episodic hypoxaemia measured by pulse oximetry may represent pathophysiological events rather than artefacts. Nevertheless, the issue of reliability of pulse oximetry after myocardial infarction with suspected high incidence of heart failure needs further study.

Patients with myocardial infarction may have a disturbed sleep pattern with a primary depression in REM sleep followed by an apparent rebound increase in REM sleep until the ninth night after myocardial infarction, ${ }^{15}$ with a potential increase in sleep disordered breathing and episodes of apnoea. Sleep apnoea may be central, due to a neurological disturbance with a cessation in respiratory activity, obstructive, due to occlusion of the upper airways, or have mixed causes. ${ }^{16}$ The number of apnoea episodes required for the sleep apnoea syndrome to be diagnosed varies from 5 apnoeas per hour ${ }^{17} 18$ to 10 apnoeas per hour. ${ }^{1920}$ Gould et al have suggested that episodes of hypopnoea with arterial oxygen desaturation are equally clinically important, they summed episodes of apnoea and hypopnoea and chose 30 per night as the threshold for a diagnosis of the sleep apnoea syndrome. ${ }^{21}$

As we did not monitor respiratory movements but knew that apnoea in the sleep apnoea syndrome is usually associated with hypoxaemia, ${ }^{21}$ we chose 30 episodes of oxygen desaturation per night as a threshold for suspected apnoea syndrome. We are aware, however, that our patients may well have developed sleep apnoea or episodic hypoxaemia as a result of the infarction with resulting heart failure, instead of having had apnoeas present before having been admitted. Because of the design of the study, however, we have not been able to pursue this hypothesis. According to the definition of 30 desaturations per night as a threshold, we found an incidence of suspected sleep apnoea syndrome varying from $6 \%$ on the second night after infarction to around $20 \%$ on the following nights. This suggests a rise in the number of episodic desaturations in the later stage after acute myocardial infarction and a higher incidence of suspected sleep apnoea among patients with myocardial infarction than among controls, in whom the incidence has been reported to be between $4 \%^{6}$ and $10-15 \%$ in unselected middle aged people. ${ }^{16}$ Although our study was small and adjusted for factors leading to sleep apnoea, our finding is consistent with that of Hung et al who found sleep apnoea in 36 out of 101 men after myocardial infarction when they used an apnoea index of 5 per hour. ${ }^{6}$ On the first night of monitoring we found a lower incidence of suspected sleep apnoea, which might be explained by the depression in REM sleep after trauma among several of our patients. We found prolonged episodes of oxygen saturation below $85 \%$ in three patients (cases 4,9 , and 15) only during the first night of monitoring.

Coincidence between arrhythmias, ischaemia, and hypoxaemia has been found in patients with chronic left heart failure ${ }^{23}$ and after operation ${ }^{11}$ and between arrhythmias and hypoxaemia in patients with myocardial infarction. ${ }^{7}$ None of the patients had sustained life threatening arrhythmias. ${ }^{237}$ Hypoxaemia was temporally associated with ischaemia (fig 2) and arrhythmias (figs 3 and 4). The occurrence of arrhythmias after acute myocardial infarction predicts a poorer prognosis $^{22}$ and experimental studies have suggested that repeated episodes of ischaemia may have a cumulative effect and eventually cause myocardial infarction. ${ }^{23}$

We chose nocturnal monitoring because we expected more episodic hypoxaemia during the night. After myocardial infarction sleep is disturbed, with a decrease in REM sleep and a subsequent rebound. ${ }^{15}$ Such a sleep disturbance with rebound of REM sleep bears a risk of an increase in apnoeas and episodic hypoxaemia as seen after abdominal surgery. ${ }^{24}$ Whether episodic hypoxaemia is causally related to arrhythmias and ischaemia, or both (reflecting, for example, brainstem hypoperfusion and autonomic instability) is not known, but the association between electrocardiographic abnormalities and hypoxaemia after acute myocardial infarction is interesting and requires further study.

Treatment of patients with acute myocardial infarction after the acute stage have mostly focused on reducing oxygen demand, but the question of increasing oxygen supply has been more or less neglected. Previous studies of oxygen treatment after acute myocardial infarction have suggested an apparent benefit in reducing the infarct size, ${ }^{25}$ and an ischaemic injury estimated from the sum of ST elevations was reduced in patients when supplemental oxygen was given. ${ }^{26}$ In patients with congestive heart failure both supplemental oxygen ${ }^{27}$ and application of continuous positive airway pressure ${ }^{28}$ reduced the number of episodic oxygen desaturations, just as in patients with a sleep apnoea syndrome. ${ }^{29}$ 
Only a few of our patients received supplemental oxygen, but because the study was not controlled for oxygen treatment we were not able to evaluate the influence of oxygen on arterial oxygenation after infarction. Controlled studies of oxygen treatment and oximetric monitoring of patients after acute myocardial infarction are warranted to rationalise oxygen treatment in such patients.

In conclusion, we found that patients have both episodic and constant hypoxaemia and that this is often associated with electrocardiographic abnormalities on the second to the sixth nights after acute myocardial infarction. Episodic and constant nocturnal hypoxaemia may be detrimental to the infarcted myocardium, and special attention should therefore be directed towards nocturnal hypoxaemia in the early phase after infarction. Further studies are needed to show to what extent routine monitoring and oxygen treatment would be beneficial and to evaluate the possible causal relation between hypoxaemia and electrocardiographic abnormalities.

1 Montague TJ, Ikuta RM, Wong RY, Bay KS, Teo KK, Davies NJ. Comparison of risk patterns of practice in patients older and younger than 70 years with acute patients older and younger than 70 years with acute
myocardial infarction in a 2-year period (1987-1989). myocardial infarction in a 2 -

2 Cripps T, Rocker G, Stradling J. Nocturnal hypoxia and arrhythmias in patients with impaired left ventricula function. Br Heart F 1992;68:382-6.

3 Davies S, John L, Wedzicha J, Lipkin D. Overnight studies in severe chronic left heartfailure: arrhythmias and oxygen desaturation. Br Heart f 1991;65:77-83.

4 Trask C, Cree E. Oximeter studies on patients with chronic obstructive emphysema, awake and during sleep. N Engl f Med 1962;266:639-42.

5 Philipson EA, Goldstein RS. Breathing during sleep in patients with chronic obstructive pulmonary disease. Chest 1984;85:24-30.

6 Hung J, Whitford E, Parsons R, Hillmann D. Association of sleep apnoea with myocardial infarction in men. Lance 1990;336:261-4.

7 Toshiyuki S, Tatsusuke Y, Yujiro S, Keiji T, Tetsuo I, Ryo . Sleep apnea in patients with acute myocardial infarction. Crit Care Med 1991;19:938-41.

8 Tilkian A, Guilleminault C, Schroeder J, Stuart J. Sleep induced apnoea syndrome. Prevalence of cardiac arrhthmias and their reversal after tracheostomy. $A m \mathcal{F} \mathrm{Med}$ 1977;63:348-58.

9 Guilleminault C, Connolly S, Winkle R. Cardiac arrhyth- mias and conduction disturbances during sleep in 400 patients with sleep apnea syndrome. Am $\mathcal{F}$ Cardiol 1983; 52:490-4

10 Tremper KK, Barker SJ. Pulse oximetry. Anesthesiology 1989;70:98-108.

11 Rosenberg J, Rasmussen V, Von Jessen F, Ullstad T, Kehlet $\mathrm{H}$. Late postoperative episodic and constant hypoxaemia and associated ECG-abnormalities. $\mathrm{Br} \mathcal{F}$ Anaesth 1990;65:684-91.

12 Jespersen CM, Rasmussen V. Value of a bipolar modified inferior lead in detection of inferior myocardial ischaemia. Br Heart $\mathcal{F} 1988 ; 60: 287-9$.

13 Langhton JA, Hanning CD. Effect of motion artefact on pulse oximeters: evaluation of four instruments and finger pulse oximeters: evaluation of four instrun

14 Braghiroli A, Sacro C, Carone M, Donner CF. Pulse oximeter and transcutaneous $\mathrm{O}_{2}$ monitoring: criteria for a choice. Eur Respir $¥$ 1990;3(suppl 11):515-6s.

15 Broughton R, Baron R. Sleep patterns in the intensive care unit and on the ward after acute myocardial infarction. Electroenceph Clin Neurophysiol 1978;45:348-60.

16 Jennum P, Sørensen PS. Sleep apnoea. Nord Med 1989; 104:250-3

17 Berry DT, Webb WB, Block AJ. Sleep apnea syndrome: a critical review of the apnea index as a diagnostic criterion. Chest 1984;86:529-31.

18 Sanders MH. Nasal CPAP effect on pattern of sleep apnea. Chest 1984;86:839-44.

19 Lavie P, Ben-Josef R, Ruben A. Prevalence af sleep apnea syndrome among patients with essential hypertension. Am Heart $\mathcal{f} 1984$;108:373-6.

20 Fletcher EC, DeBehnke RD, Lovoi MS, Gorin AB Undiagnosed sleep apnea in patients with essential hypertension. Am Heart $\mathcal{f} 1985 ; 103: 190-5$.

21 Gould GA, Whyte GB, Airlie MA, Cateral JR, Shapiro CM, Douglas NJ. The sleep apnea syndrome. Am Rev Respir Dis 1988;137:895-8.

22 Møller M, Pindborg T, Sandøe E, Simonsen EH, Thomsen PEB. Cardiac arrhythmias. Ugeskr Laeger 1992;154:700-14.

23 Geft IL, Fishbein MC, Ninomiya K, Hashida J, Chaux E, Yano J, et al. Intermittent brief periods of ischemia have cumulative effects and may cause myocardial necrosis. Circulation 1982;66:1150-3

24 Rosenberg J, Wildschiødtz G, Pedersen MH, Von Jessen F, Kehlet $H$. Late postoperative nocturnal episodic hypoxaemia and associated sleep pattern. $\mathrm{Br} \mathcal{F}$ Anaesth (in press)

25 Maroko P, Radvany P, Braunwald E, Hale S. Reduction of infarct size by oxygen inhalation following acute myocardial coronary occlusion. Circulation 1975;52:360-8.

26 Madias J, Madias N, Hood W. Precordial ST-segment mapping. Effect of oxygen inhalation on ischemic injury in patients with acute myocardial infarction. Circulation 1976;53:411-7.

27 Hanly PJ, Millar TW, Steljes DG, Baert R, Frais MA Kryger $M H$. The effect of oxygen on respiration and leep in patients with congestive heart failure. Ann Intern Med 1989;111:777-82.

28 Takasaki Y, Orr D, Popkin J, Rutherford R, Liu P, Bradley $T$. Effect of nasal continuous positive airway pressure on sleep apnea in congestive heart failure. Am Rev Respir Dis 1989;140:1578-84.

29 Brown I, McClean P, Boucher R, Zamel N, Hoffstein V. Changes in pharyngeal cross-sectional area with posture and application of continuous positive airway pressure in patients with obstructive sleep apnoea. Am Rev Respir Dis 1987;136:628-32. 